\title{
Ocean mixing processes (OMIX): impact on biogeochemistry, climate and ecosystem
}

\author{
Ichiro Yasuda ${ }^{1}$. Shuhei Masuda ${ }^{2}$ Jun Nishioka ${ }^{3}$ Xinyu Guo ${ }^{4}$ Naomi Harada ${ }^{2}$. Shin-ichi Ito ${ }^{1}$. Toshiyuki Hibiya ${ }^{5}$. \\ Hiroyasu Hasumi ${ }^{1}$
}

Published online: 18 November 2020

(c) The Oceanographic Society of Japan and Springer Nature Singapore Pte Ltd. 2020

This special section of Journal of Oceanography is a collection of papers from a project "Ocean mixing processes (OMIX): impact on biogeochemistry, climate and ecosystem (2015-2019)" funded as MEXT Kakenhi of the Grant-in-Aid for Scientific Research on Innovative Areas. OMIX had been organized by 8 primary teams with about 70 scientists with 2-year 30 selected members. The central issue of OMIX is the ocean diapycnal (vertical) mixing, which is a fundamental process that regulates ocean overturning (meridionaldiapycnal) circulations of water, nutrients, carbon and heat. However, its distribution and generation mechanisms have not been well known because of the difficulties of observations. OMIX attempted to expand integrated mixing and biogeochemical studies by developing efficient mixing measurements and elucidating turbulent nutrient fluxes to maintain ecosystem in the Oyashio and the Kuroshio and their originating regions. This leads to findings: tide-induced enhanced turbulent mixing around islands and near rough topography and the Kuroshio-topography interactions play essential roles in uplifting iron and nitrate in the Oyashio and Kuroshio regions, respectively, those of which support the active biological production around Japanese waters. The mixing observations were synthesized by a data assimilation

Ichiro Yasuda

ichiro@aori.u-tokyo.ac.jp

1 Atmosphere and Ocean Research Institute, The University of Tokyo, Kashiwa, Chiba, Japan

2 Japan Agency for Marine-Earth Science and Technology, 2-15, Natsushima, Yokosuka, Kanagawa 237-0061, Japan

3 Institute of Low Temperature Science, Hokkaido University, Sapporo, Hokkaido, Japan

4 Center for Marine Environmental Studies, Ehime University, Matsuyama, Ehime, Japan

5 Graduate School of Science, The University of Tokyo, Hongo, Tokyo, Japan approach to show an aspect of global mixing distribution and to improve quantification of deep water warming and biogeochemical cycles. Knowledge from the observations are also included in the next-generation numerical models with mixing variability to elucidate three-dimensional deep circulation in the North Pacific, which also could affect global climate through sea-ice distribution. Bi-decadal Ocean and climate variability caused by 18.6 -year period tidal mixing modulation were also studied with observations and high-resolution climate models with mixing variability. To improve the knowledge of fish reproduction processes, fish-otholith oxygen isotope analysis methods were developed to help finding the favorable environmental conditions and migration routes for the recruitment of chub-mackerel, the conditions which could be related to enhanced mixing and its variability. OMIX, a new interdisciplinary study on ocean mixing, opened new integrated sciences from physical, chemical, biological oceanography to climate and fisheries sciences. More than 100 integrated mixing and bio-geochemical expeditions including in the foreign waters by the collaboration with Russia, Indonesia, Philippine, China and USA, and over 2000 casts were performed under OMIX, and more than 400 papers were published (see OMIX web-site https://omix.aori.u-tokyo.ac.jp/achievements/). This OMIX section consists of collection of original articles and reviews synthesizing new findings published in other journals. We hope this OMIX collection could foster the new generation scientists to develop further elucidation of ocean and climate systems. We thank the Oceanographic Society of Japan for providing an opportunity to publish this special section, especially to Professor Naoto Ebuchi, Editor-in-Chief of the Journal of Oceanography. 